\title{
CUMARINAS E ALCALOIDES DE Rauia resinosa (RUTACEAE)
}

\author{
Tatiane Regina Albarici, Paulo Cezar Vieira*, João Batista Fernandes e Maria Fátima das Graças Fernandes da Silva \\ Departamento de Química, Universidade Federal de São Carlos, CP 676, 13560-970 São Carlos - SP, Brasil \\ José Rubens Pirani \\ Departamento de Botânica, Instituto de Biociências, Universidade de São Paulo, Rua do Matão, 277, 05508-900 São Paulo - SP, Brasil
}

Recebido em 3/7/10; aceito em 29/9/10; publicado na web em 8/11/10

\begin{abstract}
COUMARINS AND ALKALOIDS FROM Rauia resinosa (RUTACEAE). The genus Rauia, that is poorly chemically studied, belongs to the Rutaceae family. This family has been known to contain a large variety of secondary metabolites. Our phytochemical investigation of the stem and leaves of Rauia resinosa has led to the identification of the structurally related coumarins: murralongin (1), murrangatin (2), munomicrolin (3), murrangatin diacetate (4), umbelliferone (5), rauianin (6) and one novel coumarin: 3-ethylrauianin (7); the alkaloids: $N$-methyl-4-methoxy-2-quinolone (8), mirtopsine (9), dictamine (10), $\gamma$-fagarine (11), skimmianine (12), Z-dimethylrhoifolinate (13), zantodioline (14), zantobungeanine (15), veprissine (16), one novel alkaloid 7-hydroxy-8-methoxy$N$-methylflindersine (17) and 8-hydroxy- $N$-methylflindersine (18) that is described as a natural product for the first time, and a mixture of steroids: as sitosterol and stigmasterol.
\end{abstract}

Keywords: Rauia resinosa; coumarins; alkaloids.

\section{INTRODUÇÃO}

O gênero Rauia, taxonomicamente posicionado na família Rutaceae, subfamília Rutoideae, tribo Galipeae (anteriormente Cusparieae) e sub-tribo Galipeinae (antes Cuspariinae), ${ }^{1,2}$ ainda é pouco conhecido com relação ao seu potencial químico. De Rauia resinosa Nees \& Mart foi isolada a cumarina conhecida como rauianina. ${ }^{3}$

A família Rutaceae é constituída de aproximadamente 150 gêneros e 1600 espécies de árvores e arbustos distribuídas nas regiões tropicais e temperadas do globo terrestre. ${ }^{4}$ No Brasil estão citados 33 gêneros e aproximadamente 192 espécies. ${ }^{5}$ Esta família é conhecida por apresentar uma grande diversidade de metabólitos secundários, destacando-se os alcaloides, especialmente os derivados do ácido antranílico, cumarinas, lignanas, flavonoides, terpenos e limonoides com largo espectro de atividades biológicas. ${ }^{4,6,7}$

As cumarinas são substâncias amplamente encontradas em plantas, entretanto, espécies pertencentes às famílias Rutaceae e Umbelliferae representam as fontes mais ricas destes metabólitos, onde ocorrem com grande diversidade estrutural. ${ }^{8,9}$

Os alcaloides relatados na ordem Rutales são derivados do ácido antranílico, triptofano, fenilalanina, histidina, ácido nicotínico, ornitina ou lisina e, em alguns casos, podem ser derivados de dois precursores. ${ }^{4}$ Os alcaloides derivados do ácido antranílico têm distribuição restrita fora da família Rutaceae, atuando como marcador quimiotaxonômico desta. ${ }^{10}$

\section{PARTE EXPERIMENTAL}

\section{Procedimentos gerais}

Os espectros de ressonância magnética nuclear de hidrogênio e carbono-13 (RMN ${ }^{1} \mathrm{He} \mathrm{e}^{13} \mathrm{C}$ ) em uma e duas dimensões foram obtidos nos aparelhos ARX $200 \mathrm{MHz}$ e DRX $400 \mathrm{MHz}$ da Bruker. Os solventes utilizados foram $\mathrm{CDCl}_{3}$ e $\mathrm{CD}_{3} \mathrm{OD}$ e TMS como padrão interno.

*e-mail: paulo@dq.ufscar.br

\#Artigo em homenagem ao Prof. Hans Viertler
As análises cromatográficas em camada fina foram realizadas em cromatoplacas de sílica gel $\mathrm{F}_{254}$ sobre placa de alumínio Merck, de 0,2 mm de espessura, empregando-se como revelador solução de vanilina/ ácido sulfúrico. As separações cromatográficas em colunas foram realizadas utilizando-se gel de sílica 60, 70-230 e 230-400 mesh. As separações por cromatografia líquida de alta eficiência (CLAE) em condições preparativas foram realizadas em coluna Shodex Asahipak GS-310 2G (2,15 x 50,0 cm), utilizando metanol como fase móvel e fluxo de $7 \mathrm{~mL} / \mathrm{min}$. O equipamento utilizado foi SCL10A $v p$ Shimadzu, com válvula de injeção Rheodyne 7725i, bomba LC 6AD Shimadzu e detector de ultravioleta modelo SPD 10AV $v p$ Shimadzu em dois comprimentos de onda simultaneamente, 365 e $254 \mathrm{~nm}$.

Para a cromatografia em camada delgada preparativa (CCDP) foram utilizadas placas de vidro de $20 \times 20 \mathrm{~cm}$ e para a cromatografia em camada delgada preparativa rotativa (CCDPR) foram utilizadas placas de vidro de $26 \mathrm{~cm}$ de diâmetro, ambas utilizando como fase estacionária sílica gel $60 \mathrm{~F}_{254}$ - Merck, preparadas com espessura da camada de 1,5 mm. A CCDPR foi realizada em aparelho Cromatotrom modelo 8924-Harrison Research, utilizando bomba modelo RHSY- Fluid Metering inc.

As análises por espectrometria de massas de alta resolução foram realizadas em VG- AutoSpec a $70 \mathrm{eV}$.

\section{Material botânico}

O material botânico foi coletado e devidamente identificado pelo Prof. Dr. J. R. Pirani do Departamento de Botânica, Instituto de Biociências, Universidade de São Paulo, onde se encontra depositada uma exsicata (número de herbário 38274).

\section{Obtenção dos extratos}

Os extratos das folhas e do caule de Rauia resinosa foram preparados a partir da adição de solventes orgânicos em ordem crescente de polaridade, hexano, diclorometano e metanol, respectivamente, ao material vegetal seco e moído. Para cada solvente adicionado foram feitas 3 extrações em um intervalo de no mínimo 2 dias. Os extratos foram concentrados por destilação de solventes sob pressão reduzida. 
Os extratos metanólicos do caule e das folhas foram inicialmente submetidos à partição líquido-líquido através da suspensão em uma mistura de água destilada e $25 \%$ de metanol $(v / v)$ e transferidos para um funil de separação de $1 \mathrm{~L}$. Em seguida, foi adicionado hexano e após agitação e formação de fase a fração hexânica foi separada. Posteriormente, o procedimento foi repetido adicionando-se diclorometano e acetato de etila, respectivamente, à fase metanol-água restante. As fases orgânicas obtidas foram concentradas por destilação do solvente sob pressão reduzida em evaporadores rotativos.

\section{Isolamento dos constituintes químicos do extrato metanólico do caule de Rauia resinosa}

O extrato metanólico do caule de Rauia resinosa (103,0 g) (RCM) foi submetido à partição líquido-líquido, gerando quatro frações: hexânica (RCMH), diclorometânica (RCMD), acetato de etila (RCMA) e hidroalcoólica (RCM-hidro)

A fração $\mathrm{RCMH}(3,0 \mathrm{~g})$ foi, inicialmente, submetida à $\mathrm{CC}$ utilizando-se sílica gel (70-230 mesh) eluída com hex/AcOEt (15$100 \%$ ) em gradiente de polaridade. Em seguida, as frações foram submetidas a vários fracionamentos por CC, utilizando-se sílica gel (230-400 mesh) eluída com hex/AcOEt (5-100\%) em gradiente de polaridade, fornecendo a substância $8(26,0 \mathrm{mg})$ e a mistura dos esteroides sitosterol e estigmasterol $(65,1 \mathrm{mg})$.

A fração RCMD (10,5 g) foi submetida à CC, utilizando-se sílica gel (70-230 mesh) eluída com $\mathrm{CH}_{2} \mathrm{Cl}_{2} / \mathrm{MeOH}$ (2-100\%) em gradiente crescente de polaridade, gerando 13 frações. A eluição com $\mathrm{CH}_{2} \mathrm{Cl}_{2} / \mathrm{MeOH}$ $10,15,20$ e $30 \%$ levou à obtenção das frações $2,3,4$ e 6 , respectivamente.

A fração RCMD 2 (1,0 g) foi submetida vários fracionamentos por CC utilizando sílica gel (230-400 mesh) eluída com hex/AcOEt (5-100\%) e AcOEt/MeOH (0-100\%), fracionamentos por CC utilizando sílica gel (230-400 mesh) eluída com $\mathrm{CH}_{2} \mathrm{Cl}_{2} / \mathrm{MeOH}(2-100 \%)$ em gradiente crescente de polaridade, levando ao isolamento das substâncias 1 (1,0 mg) e $\mathbf{6}$ (368 mg). As sub-frações RCMD 2.4 (106,9 mg) e RCMD 2.5 (500 mg) foram ainda submetidas à CCDP utilizando como eluentes: $\mathrm{CH}_{2} \mathrm{Cl}_{2}$ levando ao isolamento das substâncias $\mathbf{1 5}$ (2,7 mg) e $\mathbf{1 6}$ (4,1 mg); $\mathrm{CH}_{2} \mathrm{Cl}_{2} / \mathrm{MeOH}$ na proporção 98:2 levando ao isolamento das substâncias $\mathbf{5}(2,9 \mathrm{mg})$ e $\mathbf{1 0}(1,6 \mathrm{mg})$ e hex/AcOEt levando ao isolamento das substâncias 3 (1,0 mg), 4 (1,0 $\mathrm{mg})$ e $\mathbf{1 3}(2,3 \mathrm{mg})$ e à CLAE preparativa eluída com $\mathrm{MeOH}$ levando ao isolamento da substância $7(1,0 \mathrm{mg})$.<smiles>[2H]c1c(OC)ccc2ccc(=O)oc12</smiles><smiles>CC(C)=C(C)C=O</smiles><smiles>C=C(C)[C@H](O)[C@H](O)[C@@H](C)O</smiles><smiles>C=C(C)[C@H](O)C(C)(C)C(C)(C)C</smiles><smiles>C=C(C)C(OC(C)=O)C(CC)OC(C)=O</smiles>

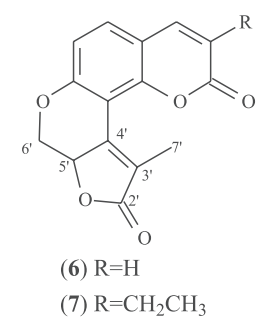

Figura 1. Cumarinas isoladas de Rauia resinosa
A fração RCMD 3 (884,0 mg) foi submetida a vários fracionamentos por CC utilizando sílica gel (230-400 mesh) eluída com hex/ $\mathrm{CH}_{2} \mathrm{Cl}_{2}$ (50-100\%), $\mathrm{CH}_{2} \mathrm{Cl}_{2} / \mathrm{MeOH}(0-100 \%$ ) e hex/AcOEt (30-100\%) em gradiente crescente de polaridade, levando ao isolamento das substâncias $\mathbf{2}(14,7 \mathrm{mg}), \mathbf{1 1}(3,2 \mathrm{mg}), \mathbf{1 2}(2,2 \mathrm{mg})$ e $\mathbf{1 7}(10,0 \mathrm{mg})$. A subfração RCMD 3.6 (640 mg) foi ainda submetida à CCDP utilizando como eluente: $\mathrm{CH}_{2} \mathrm{Cl}_{2} / \mathrm{MeOH}$ na proporção 98:2 levando ao isolamento das substâncias $\mathbf{2}(3,8 \mathrm{mg})$ e $\mathbf{8}(35,5 \mathrm{mg})$ e na proporção 96:4 que levou ao isolamento das substâncias $3(6,6 \mathrm{mg}), \mathbf{8}(7,6 \mathrm{mg})$, $12(4,4 \mathrm{mg})$ e $\mathbf{1 7}(2,8 \mathrm{mg})$ e hex/AcOEt na proporção 1:1 levando ao isolamento das substâncias $\mathbf{3}(9,8 \mathrm{mg})$ e $\mathbf{1 7}(4,6 \mathrm{mg})$.

A fração RCMD $4(660,0 \mathrm{mg})$ foi submetida a vários fracionamentos por CC utilizando sílica gel (230-400 mesh) eluída com $\mathrm{CH}_{2} \mathrm{Cl}_{2} / \mathrm{MeOH}$ (2-100\%) em gradiente crescente de polaridade. As subfrações RCMD 4.4 (39,8 mg) e RCMD 4.5 (351,0 mg) foram ainda submetidas à CCDP utilizando como eluentes: $\mathrm{CH}_{2} \mathrm{Cl}_{2} / \mathrm{MeOH}$ na proporção 96:4 levando ao isolamento da substância $\mathbf{8}(2,5 \mathrm{mg}) \mathrm{e}$ hex/AcOEt na proporção 1:1 levando ao isolamento das substâncias $\mathbf{2}(1,5 \mathrm{mg}), \mathbf{1 8}(2,5 \mathrm{mg})$ e $\mathbf{1 4}(1,0 \mathrm{mg})$.

A fração RCMD $6(676,0 \mathrm{mg})$ foi submetida a vários fracionamentos por CC utilizando como fase estacionária Sephadex LH-20 eluída com MeOH e utilizando sílica gel (230-400 mesh) eluída com $\mathrm{CH}_{2} \mathrm{Cl}_{2} / \mathrm{MeOH}$ (2-100\%) em gradiente crescente de polaridade. A subfração RCMD $6.3(47,1 \mathrm{mg})$ foi ainda submetida à CLAE preparativa, levando ao isolamento das substâncias $8(13,4)$ e $9(2,5 \mathrm{mg})$.

\section{Isolamento de compostos do extrato metanólico das folhas de Rauia resinosa}

O extrato metanólico das folhas de Rauia resinosa (RFM) foi submetido à partição líquido-líquido seguindo o procedimento descrito no item anterior, gerando quatro frações: hexânica (RFMH), diclorometânica (RFMD), acetato de etila (RFMA) e hidroalcoólica (RFM-hidro).

A RFMD (4,8 g) foi inicialmente submetida à $\mathrm{CC}$ utilizando sílica gel (70-230 mesh) eluída com hex/ $\mathrm{CH}_{2} \mathrm{Cl}_{2}(5-100 \%)$ e $\mathrm{CH}_{2} \mathrm{Cl}_{2}$ / $\mathrm{MeOH}$ (2-100\%) em gradiente crescente de polaridade, gerando 10 frações. As frações RFMD 4 (291,0 mg) e RFMD 5 (291,0 mg) foram submetidas à CCDPR eluída com hex $/ \mathrm{CH}_{2} \mathrm{Cl}_{2}(50-100 \%) \mathrm{e}$ $\mathrm{CH}_{2} \mathrm{Cl}_{2} / \mathrm{MeOH}(0-50 \%)$, levando ao isolamento das substâncias 5 $(38,9 \mathrm{mg})$ e $6(60,8 \mathrm{mg})$.

\section{3-etilrauianina (7)}

Sólido branco. EMARIE $\mathrm{m} / z, \mathrm{M}^{+}$298,0845; calculado para: 298,0814; $\mathrm{C}_{17} \mathrm{H}_{14} \mathrm{O}_{5}$. $\mathrm{m} / \mathrm{z}$ (intensidade relativa \%): 298,0845 (100), $269,0805\left[\mathrm{M}-\mathrm{CH}_{2} \mathrm{CH}_{3}\right]$ (33).

\section{7-hidroxi-8-metoxi-N-metilflindersina (17)}

Sólido branco. EMARIE $\mathrm{m} / z$ [M] $]^{+} 287,1146$; calculado para: 289, 1314; $\mathrm{C}_{16} \mathrm{H}_{17} \mathrm{NO}_{4} . \mathrm{m} / z$ (intensidade relativa \%): 287,1146 (31), $272,0915\left[\mathrm{M}-\mathrm{CH}_{3}\right](100)$.

\section{8- hidroxi-N-metilflindersina (18)}

Sólido branco. EMARIE $\mathrm{m} / \mathrm{z}$ [M] ${ }^{+} 257,1011$; calculado para: 259,1208; $\mathrm{C}_{15} \mathrm{H}_{15} \mathrm{NO}_{3} . \mathrm{m} / \mathrm{z}$ (intensidade relativa \%): 257,1011 (30), $242,0804\left[\mathrm{M}-\mathrm{CH}_{3}\right](100)$.

\section{RESULTADOS E DISCUSSÃO}

Do caule de Rauia resinosa foram isoladas sete cumarinas, onze alcaloides e dois esteroides e das folhas foram isoladas duas cumarinas. 
<smiles>COc1cc(=O)n(C)c2ccccc12</smiles>

(8)<smiles>[R]c1ccc2c(OC)c3ccoc3nc2c1[18OH]</smiles><smiles>COC(=O)/C=C\c1c(C(=O)O)nc2occc2c1OC</smiles>

(13)<smiles>[R]c1ccc2c3c(c(=O)n(C)c2c1Br)C=CC(C)(C)O3</smiles><smiles>COc1c2c(nc3ccccc13)OC(C(C)(C)C)C2O</smiles>

(9)

\section{(10) $\mathrm{R}=\mathrm{R}_{1}=\mathrm{H}$}

(11) $\mathrm{R}=\mathrm{H} \mathrm{R}_{1}=\mathrm{OCH}_{3}$

(12) $\mathrm{R}=\mathrm{R}_{1}=\mathrm{OCH}_{3}$<smiles></smiles>

(14)

(15) $\mathrm{R}=\mathrm{H} \mathrm{R}_{1}=\mathrm{OCH}_{3}$

(16) $\mathrm{R}=\mathrm{R}_{1}=\mathrm{OCH}_{3}$

(17) $\mathrm{R}=\mathrm{OH} \mathrm{R} \mathrm{R}_{1}=\mathrm{OCH}_{3}$

(18) $\mathrm{R}=\mathrm{H} \mathrm{R}_{1}=\mathrm{OH}$
Figura 2. Alcaloides isolados de Rauia resinosa

Da fração hexânica proveniente do extrato metanólico do caule de Rauia resinosa (RCMH) foram isolados o alcaloide $N$-metil4-metoxi-2-quinolona (8) e a mistura dos esteroides sitosterol e estigmasterol.

Da fração diclorometânica do extrato metanólico do caule de Rauia resinosa (RCMD) foram isoladas as cumarinas murralongina
(1), murrangatina (2), munomicrolina (3), acetato de murrangatina (4), umbeliferona (5), rauianina (6) e 3-etilrauianina (7) inédita na literatura. Foram isolados também onze alcaloides: $N$-metil-4metoxi-2-quinolona (8), mirtopsina (9), dictamina (10), fagarina (11), esquimianina (12), Z-rhoifolinato de dimetila (13), zantobungeanina (14), zantodiolina (15), veprissina (16), 7-hidroxi-8-metoxi- $N$-metilflindersina (17) inédita na literatura e a 8-hidroxi- $N$-metilflindersina (18) relatada pela primeira vez como produto natural.

Os espectros de RMN ${ }^{1} \mathrm{H}$ das substâncias 1-4 e 6 apresentaram o mesmo padrão de sinais na região aromática, sendo quatro dubletos integrando para um hidrogênio cada, característicos de sistema de acoplamento AX. Dois deles em $\delta 6,1-6,4$ e $\delta 7,5-8,3$, com constante de acoplamento da ordem de $9,5 \mathrm{~Hz}$, referentes aos hidrogênios nas posições 3 e 4 , respectivamente, e outros dois com constante de acoplamento da ordem de $8,5 \mathrm{~Hz}$ ocorrendo em $\delta$ 6,6-6,9 e em $\delta$ 7,1-7,5, que sugerem a presença de esqueleto cumarínico com substituições nos carbonos 7 e $8 .{ }^{11}$

Os espectros de $\mathrm{RMN}{ }^{13} \mathrm{C}$ destas cumarinas apresentaram sinais característicos para o esqueleto cumarínico: $\delta$ 160,8-160,0 (C-2); $\delta$ 113,6-112,8 (C-3); $\delta 143,9-143,3$ (C-4); $\delta 113,6-111,8$ (C-4a); $\delta 130-$ $126,6(\mathrm{C}-5) ; \delta 108,1-107,1$ (C-6); $\delta 161,6-159,7$ (C-7); $\delta 116,1-112,1$ (C-8); $\delta$ 154,7-152,0 (C-8a); $\delta$ 56,4-55,8 (C7-OMe). ${ }^{12}$

O conjunto de dados acima e a comparação com os dados da literatura permitiram a identificação das cumarinas murralongina ${ }^{13,14}$ (1), murrangatina ${ }^{12-15}(\mathbf{2})$, munomicrolina ${ }^{12,13,16}(\mathbf{3})$, acetato de murrangatina $^{16}(\mathbf{4})$, umbeliferona ${ }^{17}(\mathbf{5})$, rauianina ${ }^{3}(\mathbf{6})$.

A substância 7 foi isolada como um sólido branco. O espectro de $\mathrm{RMN}{ }^{1} \mathrm{H}$ mostrou sinais em $\delta 7,41$ e $\delta 6,87(J=8,6 \mathrm{~Hz})$, referentes aos hidrogênios nas posições 5 e 6 , respectivamente. A presença de 4 sinais $\delta 5,11$ (ddq, $1 \mathrm{H}, J=11,8 ; 6,0$ e $1,6 \mathrm{~Hz}$ ), $\delta 4,83$ (dd, $1 \mathrm{H}, J=10,1$ e $6,0 \mathrm{~Hz}), \delta 3,95(\mathrm{dd}, 1 \mathrm{H}, J=11,8$ e $10,3 \mathrm{~Hz})$ e $\delta 2,34(\mathrm{~s}, 3 \mathrm{H}, J=1,6$ $\mathrm{Hz}$ ) sugere a existência de um esqueleto carbocíclico semelhante ao da rauianina. A Tabela 1 mostra comparação entre os dados do espectro

Tabela 1. Dados RMN ${ }^{1} \mathrm{H}$ e $\mathrm{RMN}{ }^{13} \mathrm{C}$ de rauianina (6) e da 3-etilrauianina (7)

\begin{tabular}{|c|c|c|c|c|c|c|}
\hline & \multicolumn{2}{|c|}{ rauianina } & \multicolumn{4}{|c|}{ 3-etilrauianina } \\
\hline & \multirow[t]{2}{*}{$\delta_{\mathrm{H}}^{(\mathrm{a})}$} & \multirow[t]{2}{*}{$\delta_{\mathrm{C}}^{(b)}$} & \multirow[t]{2}{*}{$\delta_{\mathrm{H}}^{(\mathrm{a})}$} & \multirow[t]{2}{*}{$\delta_{C}^{(b)}$} & \multicolumn{2}{|c|}{ HMBC } \\
\hline & & & & & $\delta_{\mathrm{H}}\left({ }^{2} J_{\mathrm{C}-\mathrm{H}}\right)$ & $\delta_{\mathrm{H}}\left({ }^{3} J_{\mathrm{C}-\mathrm{H}}\right)$ \\
\hline 2 & & 159,5 & & $161^{*}$ & & 7,44 \\
\hline 3 & $6,35(\mathrm{~d}, 9,6 \mathrm{~Hz})$ & 113,6 & - & 128,7 & & 1,26 \\
\hline 4 & 7,73 (d, 9,6 Hz) & 143,5 & $7,44(1 \mathrm{H}, \mathrm{s})$ & 137,4 & & \\
\hline $4 a$ & & 113,0 & & 113,8 & & 6,87 \\
\hline 5 & $7,50(\mathrm{~d}, 8,6 \mathrm{~Hz})$ & 131,2 & 7,41 (d, 8,6 Hz) & 130,4 & & \\
\hline 6 & $6,92(\mathrm{~d}, 8,6 \mathrm{~Hz})$ & 113,9 & $6,87(\mathrm{~d}, 8,6 \mathrm{~Hz})$ & 113,6 & & \\
\hline 7 & & 157,3 & & $158^{*}$ & & 7,41 \\
\hline 8 & & 105,6 & & & & \\
\hline $8 \mathbf{a}$ & & 151,7 & & $152^{*}$ & & 7,$44 ; 7,41$ \\
\hline 2' & & 174,0 & & $173^{*}$ & & 2,34 \\
\hline $3^{\prime}$ & & 145,3 & & $147^{*}$ & 2,34 & \\
\hline 4 ' & & 122,9 & & $123^{*}$ & & 2,34 \\
\hline 5 & $5,15(\mathrm{ddq}, 11,8 ; 6,0 ; 1,7 \mathrm{~Hz})$ & 71,0 & $5,11(\mathrm{ddq}, 11,8 ; 6,0 ; 1,6 \mathrm{~Hz})$ & 71,1 & & \\
\hline $6^{\prime}$ & $\begin{array}{c}\text { 4,85 (dd, 10,3; 6,0 Hz) } \\
3,95(\mathrm{dd}, 11,8 ; 10,3 \mathrm{~Hz})\end{array}$ & 69,5 & $\begin{array}{c}4,83(\mathrm{dd}, 10,1 ; 6,0 \mathrm{~Hz}) \\
3,95(\mathrm{dd}, 11,8 ; 10,1 \mathrm{~Hz})\end{array}$ & 69,5 & 5,11 & \\
\hline 7 & $2,32(\mathrm{~d}, 1,7 \mathrm{~Hz})$ & 12,5 & $2,34(\mathrm{~d}, 1,6 \mathrm{~Hz})$ & 12,6 & & \\
\hline $1 "$ & - & - & $2,60(\mathrm{q}, 7,0 \mathrm{~Hz})$ & 23,7 & & 7,44 \\
\hline $2 "$ & - & - & $1,26(\mathrm{t}, 7,0 \mathrm{~Hz})$ & 12,2 & & \\
\hline
\end{tabular}

(a) $\mathrm{CDCl}_{3}, 200 \mathrm{MHz}$; (b) $\mathrm{CDCl}_{3}, 50 \mathrm{MHz}$. *valores obtidos através da projeção de carbono do HMBC 
Tabela 2. Dados de RMN ${ }^{1} \mathrm{H}$ e $\mathrm{RMN}{ }^{13} \mathrm{C}$ de 7 -hidroxi-8-metoxi- $N$ - metilflindersina (17)

\begin{tabular}{|c|c|c|c|c|}
\hline$\underline{\mathbf{H} / \mathrm{C}}$ & $\delta_{\mathrm{C}}^{\left({ }^{(c)}\right.}$ & $\delta_{\mathrm{H}}\left({ }^{1} J_{\mathrm{C}-\mathrm{H}}\right)^{(\mathrm{a})}$ & $\delta_{\mathrm{H}}\left({ }^{2} J_{\mathrm{C}-\mathrm{H}}\right)$ & $\delta_{\mathrm{H}}\left({ }^{3} J_{\mathrm{C}-\mathrm{H}}\right)$ \\
\hline 2 & 162,6 & & & 3,90 \\
\hline 3 & 103,5 & & & 5,49 \\
\hline 4 & 155,6 & & & 6,71 \\
\hline $4 a$ & 111,5 & & & 6,91 \\
\hline 5 & 111,1 & $7,69(d, 8,8 \mathrm{~Hz})$ & & \\
\hline 6 & 120,1 & $6,91(d, 8,8 \mathrm{~Hz})$ & & \\
\hline 7 & 152,5 & & & 7,69 \\
\hline 8 & 134,4 & & & 3,$73 ; 6,91$ \\
\hline $8 a$ & 134,1 & & & 3,$90 ; 7,69$ \\
\hline 2 ' & 78,8 & & 1,$50 ; 5,49$ & 6,71 \\
\hline 3 ' & 125,5 & $5,49(d, 9,9 \mathrm{~Hz})$ & & 1,50 \\
\hline 4' & 117,8 & $6,71(d, 9,9 \mathrm{~Hz})$ & & \\
\hline $2^{\prime}\left(\mathrm{CH}_{3}\right)_{2}$ & 28,2 & $1,50(s)$ & & 1,50 \\
\hline $\mathrm{O}-\mathrm{CH}_{3}$ & 62,2 & $3,90(s)$ & & \\
\hline $\mathrm{N}-\mathrm{CH}_{3}$ & 33,2 & $3,73(s)$ & & \\
\hline
\end{tabular}

a) $\mathrm{CDCl}_{3}, 200 \mathrm{MHz}$; (b) $\mathrm{CDCl}_{3}, 50 \mathrm{MHz}$

de $\mathrm{RMN}{ }^{1} \mathrm{H}$ da substância 7 e da cumarina rauianina. ${ }^{3}$ No espectro de RMN ${ }^{1} \mathrm{H}$ da substância 7 pode ser notada a ausência de dois dupletos em $\delta 7,73$ e $\delta 6,35(J=9,6 \mathrm{~Hz})$ atribuídos aos hidrogênios nas posições 4 e 3 do esqueleto cumarínico da rauianina, assim como a presença de um simpleto em $\delta 7,44$ que sugere a presença de um grupo substituinte na posição 3. Os sinais em $\delta 2,60(\mathrm{q}, 2 \mathrm{H}, J=7,5 \mathrm{~Hz})$ e $\delta 1,26(\mathrm{t}, 3 \mathrm{H}$, $J=7,5 \mathrm{~Hz}$ ) são característicos de um grupo etila. Os dados de RMN ${ }^{13} \mathrm{C}$ e do mapa de contorno de HMBC estão expressos na Tabela 1.

O espectro de massas de alta resolução exibiu o pico do íon molecular coincidindo com o pico base em $\mathrm{m} / z, 298,0845$, estando de acordo com a fórmula molecular $\mathrm{C}_{17} \mathrm{H}_{14} \mathrm{O}_{5}$, confirmando a proposta da cumarina inédita na literatura, 3-etilrauianina para 7.

As estruturas dos alcaloides $N$-metil-4-metoxi-2-quinolona ${ }^{18}(\mathbf{8})$, mirtopsina $^{19}(\mathbf{9})$, dictamina ${ }^{20}(\mathbf{1 0})$, fagarina $^{21}(\mathbf{1 1})$, esquimianina ${ }^{22}$ (12), Z-rhoifolinato de dimetila ${ }^{23}(\mathbf{1 3})$, zantodiolina ${ }^{24}$ (14), zantobungeanina $^{25}(\mathbf{1 5})$ e veprissina ${ }^{25}$ (16) foram elucidadas através de dados de EM, RMN ${ }^{1} \mathrm{H}, \mathrm{RMN}{ }^{13} \mathrm{C}$, além da comparação com dados descritos na literatura.

Os espectros de $\mathrm{RMN}{ }^{1} \mathrm{H}$ das substâncias 15-18 mostraram sinais típicos de anel 2,2-dimetilcromeno, condensado a sistema aromático, um simpleto integrando para 6 hidrogênios na região de $\delta 1,5$ referente a duas metilas geminais e dois dupletos nas regiões de $\delta 5,50$ e 6,75 com constante de acoplamento de aproximadamente $10 \mathrm{~Hz}$ referentes a dois hidrogênios olefinicos. ${ }^{27}$

A substância 17 foi isolada como um sólido branco e o espectro de $\mathrm{RMN}{ }^{1} \mathrm{H}$, além dos sinais característicos do 2,2-dimetilcromeno, apresentou dois dupletos em $\delta$ 7,69 e 6,91 com $J=8,7 \mathrm{~Hz}$, que indica uma constante de acoplamento de hidrogênios aromáticos em relação orto. Dois simpletos integrando para 3 hidrogênios cada em $\delta 3,90$ e $\delta 3,73$ sugerem a presença de duas metilas ligadas a heteroátomos. A análise do espectro de $\mathrm{RMN}{ }^{13} \mathrm{C}$ mostrou sinal em $\delta 162,3$ referente a um carbono carbonílico, além de um sinal de carbono carbinólico em $\delta 78,7$ e um sinal em $\delta 28,2$ referente às metilas geminais do cromeno. Os sinais em $\delta 62,2$ e em $\delta 35,3$ indicam a presença de duas metilas ligadas a oxigênio e nitrogênio, respectivamente. $\mathrm{O}$ deslocamento químico do carbono da metoxila $(\delta 62,2)$ indica a presença de grupos substituintes nas posições orto.
Tabela 3. Dados de $\mathrm{RMN}{ }^{1} \mathrm{H}$ e $\mathrm{RMN}{ }^{13} \mathrm{C}$ de 8 -hidroxi- $N$-metilflindersina (18) e de 8-metoxiflindersina

\begin{tabular}{|c|c|c|c|c|}
\hline \multirow[b]{2}{*}{$\mathrm{H} / \mathrm{C}$} & \multicolumn{2}{|c|}{ 8-hidroxi- $N$-metilflindersina } & \multicolumn{2}{|c|}{ 8-metoxiflindersina } \\
\hline & $\delta_{\mathbf{H}}^{(a)}$ & $\delta_{\mathbf{C}}^{(b)}$ & $\delta_{\mathrm{H}}^{(\mathrm{a})}$ & $\delta_{\mathrm{C}}{ }^{(\mathrm{b})}$ \\
\hline 2 & & 162,3 & & 160,4 \\
\hline 3 & & 105,8 & & 106,2 \\
\hline 4 & & 155,4 & & 156,8 \\
\hline $4 \mathbf{a}$ & & 118,8 & & 115,5 \\
\hline 5 & $7,52(d d, 7,8$ e $1,7 \mathrm{~Hz})$ & 118,4 & $7,45(d d, 9$ e $2,8 \mathrm{~Hz})$ & 114,4 \\
\hline 6 & $7,05(t, 7,8 \mathrm{~Hz})$ & 122,5 & $7,09(d d, 9$ e $2,8 \mathrm{~Hz})$ & 121,4 \\
\hline 7 & $7,05(d d, 7,8$ e $1,7 \mathrm{~Hz})$ & 115,7 & $6,93(d d, 9$ e $2,8 \mathrm{~Hz})$ & 110,0 \\
\hline 8 & & 145,0 & & 145,3 \\
\hline $8 \mathbf{a}$ & & 129,4 & & 127,8 \\
\hline 2' & & 78,7 & & 78,2 \\
\hline 3' & $5,54(d, 9,9 \mathrm{~Hz})$ & 126,6 & $5,53(d, 11,0 \mathrm{~Hz})$ & 126,0 \\
\hline 4 ' & $6,75(d, 9,9 \mathrm{~Hz})$ & 117,9 & $6,70(d, 11,0 \mathrm{~Hz})$ & 117,1 \\
\hline $2^{\prime}\left(\mathrm{CH}_{3}\right)_{2}$ & $1,50(s)$ & 28,2 & $1,50(s)$ & 28,2 \\
\hline $\mathrm{O}-\mathrm{CH}_{3}$ & - & - & $3,93(s)$ & 55,9 \\
\hline $\mathrm{N}-\mathrm{CH}_{3}$ & $4,05(s)$ & 35,3 & & \\
\hline N-H & - & & $8,93(s)$ & \\
\hline
\end{tabular}

(a) $\mathrm{CDCl}_{3}, 200 \mathrm{MHz}$; (b) $\mathrm{CDCl}_{3}, 50 \mathrm{MHz}$

O mapa de contorno de HSQC mostrou correlação entre os sinais da metila em $\delta 3,90$ e o sinal em $\delta 62,2$ que indica a presença de um grupo metila ligado a um átomo de oxigênio, enquanto que o sinal em $\delta 3,73$ mostrou correlação com o sinal em $\delta 33,2$, que indica a presença de um grupo metila ligado a um átomo de nitrogênio. Os dados dos espectros de $\mathrm{RMN}{ }^{1} \mathrm{H}$ e ${ }^{13} \mathrm{C}$ assim como dos mapas de contorno de HSQC e HMBC da substancia 17 estão representados na Tabela 2 .

O espectro de massas de alta resolução exibiu o pico do íon molecular coincidindo com o pico base em $\mathrm{m} / \mathrm{z}, 287,1146$, estando de acordo com a fórmula molecular $\mathrm{C}_{16} \mathrm{H}_{17} \mathrm{NO}_{4}$, confirmando a proposta da 7-hidroxi-8-metoxi- $N$-metilflindersina para 17 , inédita na literatura.

A substância 18 foi isolada como um sólido branco. O espectro de $\mathrm{RMN}{ }^{1} \mathrm{H}$ apresenta dois dupletos em $\delta$ 6,74 e $\delta$ 5,53 (H, $J=9,9$ $\mathrm{Hz})$ e um simpleto em $\delta 1,50(6 \mathrm{H})$ que sugerem a presença de um anel 2,2-dimetilcromeno. A região aromática do espectro mostra ainda um duplo dupleto em $\delta 7,52(1 \mathrm{H}, J=7,6$ e $1,7 \mathrm{~Hz})$, um tripleto em $\delta 7,05(1 \mathrm{H}, J=7,6 \mathrm{~Hz})$ e um duplo dupleto em $\delta 7,13(1 \mathrm{H}, J=7,6 \mathrm{e}$ $1,7 \mathrm{~Hz}$ ), sugerindo a presença da um anel aromático trissubstituído. Um simpleto em $\delta 4,05(3 \mathrm{H})$ sugere a presença de uma metila ligada a heteroátomo $(\mathrm{N}$ ou $\mathrm{O})$. Este espectro mostrou sinais semelhantes aos dados encontrados na literatura para a 8-metoxiflindersina, porém com a ausência de um simpleto em $\delta 8,88(1 \mathrm{H})$, como pode ser observado na Tabela 3.

Os dados do espectro de RMN ${ }^{13} \mathrm{C}$ mostram um sinal em $\delta 162,6$ referente a um carbono carbonílico, além de um sinal de carbono carbinólico em $\delta 78,8$ e um sinal em $\delta 28,2$ referente às metilas geminais do cromeno. A ausência de um sinal próximo a $\delta 56,0$ descarta a possibilidade da presença de uma metoxila na estrutura e o sinal em $\delta 35,3$ confirma a existência de uma metila ligada a nitrogênio. A análise dos dados e as comparações com os dados da literatura da 8-metoxiflindersina ${ }^{24}$ indicam que a substância 18 é o alcaloide piranoquinolônico 8-hidroxi- $N$-metilflindersina.

O espectro de massas de alta resolução da substância 18 mostrou o pico do íon molecular com $m / z$ 257,1011 (30,01\%), que está de 
acordo com a fórmula molecular $\mathrm{C}_{15} \mathrm{H}_{15} \mathrm{NO}_{3}$ o valor calculado é $\mathrm{m} / \mathrm{z}$ 259,1208 confirmando a proposta da 8-hidroxi- $N$-metilflindersina para 18. O pico base tem $\mathrm{m} / \mathrm{z}, 242(100 \%)$ e é gerado através da perda de uma metila. Na literatura há relato da síntese desta substância, porém este alcaloide não foi isolado de fontes naturais. Os dados de RMN ${ }^{1} \mathrm{H}$ e de $\mathrm{RMN}{ }^{13} \mathrm{C}$ da substância $\mathbf{1 8}$ e da 8-metoxiflindersina estão representos na Tabela 3.

\section{MATERIAL SUPLEMENTAR}

Os espectros de RMN e EM das substâncias 7, 17 e 18 estão disponíveis em http://quimicanova.sbq.org.br, em arquivo .PDF, com acesso livre.

\section{AGRADECIMENTOS}

À Fundação de Amparo à Pesquisa do Estado de São Paulo (FAPESP), pelo financiamento do trabalho.

\section{REFERÊNCIAS}

1. Engler, A.; Syllabus der pflanzenfamilien, $12^{\text {th }}$ ed., Bornträger: Berlin, 1964.

2. Kallunki, J. A.; Pirani, J. R.; Kew Bull. 1998, 53, 257.

3. Velozo, E. S.; Oliveira, D. J.; Arruda, A. C.; Vieira, P. C.; Fernandes, J. B.; Silva, M. G. F.; Caracelli, I.; Zukerman-Spector, J.; Nat. Prod. Lett. 1997, 9, 237.

4. Waterman, P. G.; Grundon, M. F.; Chemistry and Chemical Taxonomy of the Rutales, Academic Press: London, 1983.

5. Pirani, J. R.; Groppo, M. Em Catálogo de Espécies de Plantas e Fungos do Brasil; Forzza, R. C.; Leitman, P. M.; Costa, A.; de Carvalho Jr., A. A.; Peixoto, A. L.; Walter, B. M. T.; Bicudo, C.; Zappi, D.; da Costa, D. P.; Lleras, E.; Martinelli, G.; de Lima, H. C.; Prado, J.; Stehmann, J. R.; Baumgratz, J. F. A.; Pirani, J. R.; Sylvestre, L. S.; Maia, L. C.; Lohmann, L. G.; Paganucci, L.; Silveira, M.; Nadruz, M.; Mamede, M. C. H.; Bastos, M. N. C.; Morim, M. P.; Barbosa, M. R.; Menezes, M.; Hopkins, M.; Secco, R.; Cavalcanti, T.; Souza, V. C., orgs.; Jardim Botânico do Rio de Janeiro: Rio de Janeiro, 2010, p. 1592-1600.

6. Albuquerque, B. W. P.; An. Acad. Brasil. Cienc. 1968, 40, 499.
7. Waterman. P. G.; Biochem. Syst. Ecol. 1999, 27, 395.

8. Gray, A. I.; Waterman, P. G.; Phytochemistry 1978, 17, 845.

9. Estévez-Braun, A.; González, A. G.; Nat. Prod. Rep. 1997, 14, 465.

10. Waterman, P. G.; Grundon, M. F.; Biochem. Syst. Ecol. 1975, 3, 149.

11. Murray, R. D. H.; Méndez, J.; Brown, S. A.; The Natural Coumarins: Occurrence, Chemistry and Biochemistry, $1^{\text {st }}$ ed., John Wiley \& Sons: London, 1982.

12. Suarez, L. E. C.; Monache, F. D.; Rev. Latinoamer. Quim. 1991, 22, 38

13. Ito, C.; Furukawa, H.; Ishii, H.; Ishikawa, T.; Haginiwa, J.; J. Chem. Soc. Perkin Trans. 1990, 1, 2047.

14. Kinoshita, T.; Tatara, S.; Ho, F. C.; Sankawa, U.; Phytochemistry 1996, $43,125$.

15. Kinoshita, T.; Wu, J. B.; Ho, F. C.; Phytochemistry 1989, $28,147$.

16. Das, S.; Baruah, H. R.; Sharma, R. P.; Barua, J. N.; Kulanthaivel, P.; Hertz, W.; Phytochemistry 1984, 23, 2317.

17. Kong, L. Y.; Li, Y.; Min, Z. D.; Li, X.; Zhu, T. R.; Phytochemistry 1996, $41,1423$.

18. Nayar, M. N. S.; Sutar, C. V.; Bhan, M. K.; Phytochemistry 1971, 10, 2843.

19. Hifnawy, M. S.; Vaquette, J.; Sévenet, T.; Pousset, J. L.; Cavé, A.; Phytochemistry 1977, 16, 1035.

20. Pusset, J.; Lopez, J. L.; Pais, M.; Neirabeyeh, M. A.; Veillon, J. M.; Planta Med. 1991, 57, 153.

21. Cuca, S.; Martinez, V. J. C.; Monache, F. D.; Rev. Col. Quim. 1998, 27, 23.

22. Ahond, A.; Picot, F.; Potier, P.; Poupat, C. ; Sévenet, T.; Phytochemistry 1978, 17, 166.

23. Arruda, M. S. P.; Fernandes, J. B.; Vieira, P. C.; Silva, M. F. G. F. ; Pirani, J. R.; Biochem. Syst. Ecol. 1992, 20, 173.

24. Chen, I.; Tsai, I.; Teng, C.; Chen, J.; Chang, Y.; Ko, F.; Lu, M. C.; Pezzuto, J. M.; Phytochemistry 1997, 46, 525.

25. Campbell, W. E.; Davidowitz, B.; Jackson, G. W.; Phytochemistry 1990, 29, 1303

26. Ayafor, J. F.; Sondengam, B. L.; Ngadjui, B.;Tetrahedron Lett. 1980, 21, 3293.

27. Brader, G.; Bacher, M.; Greger, H.; Hofer, O.; Phytochemistry 1996, 42, 881. 